\title{
Article \\ Optimizing the Minimum Detectable Difference of Gamma Camera SPECT Images via the Taguchi Analysis: A Feasibility Study with a V-Shaped Slit Gauge
}

\author{
Ching-Hsiu Ke ${ }^{1,2}$, Wan-Ju Liu ${ }^{1,3}$, Bing-Ru Peng ${ }^{1,4}$, Lung-Fa Pan 1,5,† and Lung-Kwang Pan 1,*, \\ 1 Department of Medical Imaging and Radiological Science, Central Taiwan University of Science and \\ Technology, Takun, Taichung 406, Taiwan, China; 108364@ctust.edu.tw (C.-H.K.); \\ ky708d@hotmail.com (W.-J.L.); a0953939793@gmail.com (B.-R.P.); lung-fa@803.org.tw (L.-F.P.) \\ 2 Department of Optometry, Central Taiwan University of Science and Technology, Takun, \\ Taichung 406, Taiwan, China \\ 3 Department of Nuclear Medicine, Taichung Tzu Chi Hospital, Buddhist Tzu Chi Medical Foundation, \\ Taichung 427, Taiwan, China \\ 4 Department of Radiology, Taichung Armed Forces General Hospital, Taichung 411, Taiwan, China \\ 5 Department of Cardiology, Taichung Armed Forces General Hospital, Taichung 411, Taiwan, China \\ * Correspondence: 1kpan@ctust.edu.tw \\ + These authors contributed equally to this work.
}

check for updates

Citation: Ke, C.-H.; Liu, W.-J.; Peng,

B.-R.; Pan, L.-F.; Pan, L.-K.

Optimizing the Minimum Detectable Difference of Gamma Camera SPECT Images via the Taguchi Analysis: A Feasibility Study with a V-Shaped Slit Gauge. Appl. Sci. 2022, 12, 2708.

https://doi.org/10.3390/

app12052708

Academic Editors: Edik U. Rafailov and Andrés Márquez

Received: 20 December 2021

Accepted: 2 March 2022

Published: 5 March 2022

Publisher's Note: MDPI stays neutral with regard to jurisdictional claims in published maps and institutional affiliations.

Copyright: (C) 2022 by the authors. Licensee MDPI, Basel, Switzerland. This article is an open access article distributed under the terms and conditions of the Creative Commons Attribution (CC BY) license (https:// creativecommons.org/licenses/by/ $4.0 /)$.

\begin{abstract}
This study tried to propose an innovated idea of solidifying the resolution of gamma camera in routine quality control and recommended a quantified index as minimum detectable difference (MDD) of gamma camera SPECT images using the Taguchi analysis and an indigenous V-shaped slit gauge. The gauge was customized to fulfill the quantitative requirement of the Taguchi analysis. The MDD among slit gauge of derived SPECT image was calculated from two overlapped peak profiles collected from a tangent slice of the V-shaped slit gauge with two nearby peaks. In particular, MDD was evaluated as minimum distance between two peak centers through the Student's $t$-test with a constant, 1.96, which indicates that two peak centers separated distant enough to create a $95 \%$ confidence level of separation. Eighteen combinations of six gamma camera scanned factors were organized according to Taguchi analysis. Accordingly, (A) collimator, (B) detector to targe distance, (C) total counts, (D) acquired energy width, (E) Matrix size, and (F) zoom of collected ROI with each of two or three levels were organized into 18 groups to collect the slit gauge images according to Taguchi $\mathrm{L}_{18}$ orthogonal array. Then, three well-trained radiologists were ranked the scanned gauge images to derive the fish-bone-plot of signal-to-noise ratio $(\mathrm{S} / \mathrm{N}, \mathrm{dB})$ and correlated ANOVA. Furthermore, the quantified MDD was proposed to verify the optimal suggestion of gamma camera scanned protocol, and obtained the MDD as 8.4, 7.9, and $7.1 \mathrm{~mm}$ for the second group of original $\mathrm{L}_{18}$ preset, conventional, and the optimal preset, respectively. Thus, the optimal preset of gamma camera was achieved in this study. The MDD proved to be a successful index in quantifying the imaging resolution of a gamma camera.
\end{abstract}

Keywords: SPECT; gamma camera; Tc-99m MDP; spatial resolution; minimum detectable difference

\section{Introduction}

To propose a solid recommendation for enhancing the spatial resolution of a gamma camera is always an interesting topic in the field of nuclear medicine. In doing so, an innovated idea of minimum detectable difference (MDD) of gamma camera SPECT (Single Photon Emission Computed Tomography) images was introduced and optimized through the Taguchi analysis and an indigenous V-shaped slit gauge in this study. A bone scan is a common examination ( $\sim 30 \%$ the work load in routine examination) in the nuclear medicine of most Taiwanese hospitals because it can provide bountiful information on local blood flow and bone metabolic activity. This fast-screening tool for whole-body bone cancer 
metastasis [1,2] is instrumental in examining the preliminary syndrome from increased blood flow or osteogenetic activity enhancement than routine X-ray diagnosis that bone calcium needs to change over $\pm 35-50 \%$ in order to cause an apparent lesion in a clinical X-ray examination $[3,4]$. Nevertheless, bone is one of the most common sites for metastases of all cancers; thus, an optimal preset of regular bone scan protocol can greatly help examine the carcinoma in the early stage from a radiological viewpoint [5-7].

Unlike the solid specification of imaging quality for CT scanned image, the criteria of an acquired image in nuclear medicine are less focused, since a gamma camera provides the hot/cold area ratio from absorbing radioactive solution in daily examination. Many researchers have developed various techniques to enhance the quality of scanned images of a gamma camera by pre-/post-processing of the acquired imaging. Oumar and Ercelebi [8] used an indigenous phantom to evaluate the integral and differential uniformity of gamma camera images for quality control. The phantom was customized by many slits or holes of various sizes, and the infused radioactive solution was Tc-99m. Robert et al. [9] adopted a commercial phantom to optimize the spatial resolution of imaging by the practical figure of merit (FOM) and theoretical simulation. Hruska et al. [10] and Dickerscheid [11] also adopted a phantom with multiple holes of various sizes to optimize the quality by justifying the contrast-to-noise ratio and collimator.

The gamma camera imaging can be optimized using Taguchi analysis in this study. The method was adopted in the radiological field for a decade. Yeh et al. [12] first applied this technique to nuclear medicine examination with a semi-quantitative phantom to optimize the imaging quality. Kittipayak et al. [13] combined the idea of MDD with a simple in-house phantom to optimize the imaging quality of head and neck diagnosis in the routine nuclear examination. The Taguchi analysis gained its high reputation in providing efficient and confident solutions with practical verification. Thus, the optimal protocol of gamma camera via Taguchi's recommendation is always robust, being supported by bountiful numerical proof. The quantified gauge is essential in verifying the optimization because the minimum detectable difference (MDD) adequately represents the spatial resolution. Other benefits of adopting MDD are also mentioned in this study.

\section{Materials and Methods}

\subsection{Taguchi Methodology}

The Taguchi methodology is an efficient technique to optimize high-quality feature systems. The specific analysis offers unique orthogonal arrays to contain large factor contributions by processing limited numbers of measurements. The optimal combinations of preset factors for gamma camera scanned imaging were isolated from the surrounding situations or other factors. An ANOVA (statistical analysis of variance) evaluated the factors that significantly affected the main variable. The $\mathrm{S} / \mathrm{N}$ and ANOVA analyses were integrated to ensure the combinations of factor in optimizing the gamma camera scan protocol $[14,15]$.

\subsection{The Orthogonal Arrays}

Unlike other optimized methods, Taguchi analysis assure both an optimal level of specific factor from a finite preset of empirical data and those factors that affect the aiming factor. The six factors of the gamma camera scan protocol contained the (A) collimator, (B) Detector to target distance (DTD), (C) total counts (TC), (D) acquired energy width (EW), (E) Matrix size of the acquired image, and (F) Zoom for specific ROI. Thus, a total of 486 $(2 \times 3 \times 3 \times 3 \times 3 \times 3)$ combinations were considered, since each of these factors would be assigned to two or three levels. Adopting the Taguchi analysis, the measurements were organized to only eighteen groups, which were collected to obtain effects in the similar confidence level as that derived from conventional analysis, via optimal processes [16]. Table 1 shows a standard $\mathrm{L}_{18}\left(2^{1} \times 3^{5}\right)$ orthogonal array recommended by Taguchi; the digits in each column indicate the practical organizations or specific levels, for the individual 
factors (A-F). Table 2 depicts the six factors (A-F) as (A) collimator, (B) DTD (C) TC, (D) EW, (E) Matrix, and (F) Zoom.

Table 1. Taguchi recommends the standard $\mathrm{L}_{18}\left(2^{1} \times 3^{5}\right)$ orthogonal array; the digits in every column indicate the practical organizations (or levels) of the special factors (A-F).

\begin{tabular}{ccccccc}
\hline \multirow{2}{*}{ Group } & & & & Factor & & \\
\cline { 2 - 7 } & A & B & C & D & E & F \\
\hline 1 & 1 & 1 & 1 & 1 & 1 & 1 \\
2 & 1 & 1 & 2 & 2 & 2 & 2 \\
3 & 1 & 1 & 3 & 3 & 3 & 3 \\
4 & 1 & 2 & 1 & 1 & 2 & 2 \\
5 & 1 & 2 & 2 & 2 & 3 & 3 \\
6 & 1 & 2 & 3 & 3 & 1 & 1 \\
7 & 1 & 3 & 1 & 2 & 1 & 3 \\
8 & 1 & 3 & 2 & 3 & 2 & 1 \\
9 & 1 & 3 & 3 & 1 & 3 & 2 \\
10 & 2 & 1 & 1 & 3 & 3 & 3 \\
11 & 2 & 1 & 2 & 1 & 1 & 1 \\
12 & 2 & 1 & 3 & 2 & 2 & 1 \\
13 & 2 & 2 & 1 & 2 & 3 & 2 \\
14 & 2 & 2 & 2 & 3 & 1 & 3 \\
15 & 2 & 2 & 3 & 1 & 2 & 1 \\
16 & 2 & 3 & 1 & 3 & 2 & 2 \\
17 & 2 & 3 & 2 & 1 & 3 & 1 \\
18 & 2 & 3 & 3 & 2 & & \\
\hline
\end{tabular}

Table 2. The gamma camera scan protocol has six factors, every factor with two or three levels as recommended by the Taguchi $\mathrm{L}_{18}$ orthogonal array. In addition, LEHR and LEGP stand for Low Energy High Resolution and Low Energy General Purpose, respectively.

\begin{tabular}{lccc}
\hline \multicolumn{1}{c}{ Factor } & Level 1 & Level 2 & Level 3 \\
\hline (A) collimator & LEHR & LEGP & \\
(B) detector to target distance (DTD) & $5 \mathrm{~cm}$ & $10 \mathrm{~cm}$ & $15 \mathrm{~cm}$ \\
(C) Total counts (TT) & $135 \mathrm{k}$ & $150 \mathrm{k}$ & $165 \mathrm{k}$ \\
(D) acquired energy width (EW) & $16 \%$ & $20 \%$ & $24 \%$ \\
(E) Matrix size & $128 \times 128$ & $256 \times 256$ & $512 \times 512$ \\
(F) Zoom of ROI & 1.00 & 1.28 & 1.50 \\
\hline
\end{tabular}

\subsection{The Analysis of Variance: ANOVA}

Taguchi recommended the use of a practical $\eta$ (loss function) to quantify the outcoming qualities and can easily be converted to a signal-to-noise $(\mathrm{S} / \mathrm{N})$ ratio. Larger $\mathrm{S} / \mathrm{N}$ values corresponded to high-quality features, and the optimal factor combinations produced the highest $\mathrm{S} / \mathrm{N}$ values [16]. Therefore, the quality feature of gamma camera collected image could be described as below through the specific V-shaped slit gauge by adopting various gamma camera scan protocols:

$$
\eta_{i}=-10 \log \left[\left(\frac{1}{Y(i)_{\text {avg }}}\right)^{2}+\left(\frac{\text { stdev }}{Y(i)_{\text {avg }}}\right)^{2}\right]
$$

where $\eta_{i}$ indicates the loss function $(\mathrm{S} / \mathrm{N}$ unit: $\mathrm{dB}$ ) of the $i$ th group. Larger values of $\eta$ are considered favored in this study because the rank of the scanned V-shaped slit gauge image is set as "higher-is-better". $S S_{\text {Total }}, S S_{\text {Factor, }} S S_{\text {error, }}$ and DoF (degree of freedom) are defined as:

$$
S S_{\text {Total }}=\left[\sum_{i=1}^{n} \sum_{j=1}^{r} y_{i j}^{2}\right]-n \times r \times \overline{\bar{y}}^{2}
$$




$$
\begin{gathered}
S S_{\text {Factor }}=\frac{n \times r}{L} \sum_{k=1}^{L}\left(\overline{y_{k}}-\overline{\bar{y}}\right)^{2} \\
S S_{\text {error }}=S S_{\text {total }}-\sum_{i=1}^{n} S S_{\text {Factor }_{i}} \\
D o F_{\text {Total }}=n \times r-1 ; D o F_{\text {Factor }}=L-1 ; D_{\text {o }} F_{\text {error }}=n \times(r-1)
\end{gathered}
$$

where $S S_{\text {Total }}$ implies the sum of squares for all of the variances, $r$ indicates the total number of trial repeats in every group, which is 9 in this study, $y_{i j}$ is the ranked grade of an image for the $i$ th group in the $j$ th trial, and $\overline{\bar{y}}$ implies the average of all of the ranked values of the slit gauge image (i.e., the theoretical value is 9.5). Nevertheless, $\overline{y_{k}}$ implies the average ranked value connected to that factor. $\mathrm{L}$ and $\mathrm{n}$ are the number of levels assigned to the specific factor and all groups, respectively. $S S_{\text {Factor }}$ indicates the sum of squares corresponding to the particular factor. Note that values of $\mathrm{n}$ and $\mathrm{L}$ are equal to eighteen and two/three, respectively. $S S_{\text {error }}$ implies the sum of squares of random errors only. $F_{\text {factor }}$, defined as the practical index of the F-test when fixing the individual factor, is indicated as:

$$
F_{\text {factor }}=\frac{S S_{\text {factor }}}{D o F_{\text {factor }}} / \frac{S S_{\text {error }}}{D o F_{\text {error }}}
$$

where $D o F_{i}$ is the number of degrees of freedom, its value was one or two for each of the six factors. The random error was defined as the deviation of the nine ranked grades given by the three radiologists in the three rounds [18 $\times(9-1)=144]$ (Equation (5)) [17].

\subsection{V-Shaped Slit Gauge and Gamma Camera}

The design of a V-shaped slit gauge ensures a connection with the revised $t$-test analysis. The zigzag array of the slit with a continuous change of slit depth provides the following gamma-ray image under scanning. Thus, the converted data matrix of derived SPECT can be evaluated for calculating the revised $t$-test index. Moreover, the data profile of SPECT can be judged channel-by-channel to ensure that two adjacent peaks satisfy the $95 \%$ confidence level according to the definition of the revised $t$-test after the conversion from the original gamma-ray imaging. Thus, the MDD can be quantified as precisely as to one-tenth $\mathrm{mm}$ rather than providing an approximate value within $0.3-0.6 \mathrm{~mm}$ range of the intrinsic spatial resolution for referring in reality [18].

Figure 1A shows the precise designation chart of this unique PMMA V-shaped slit gauge $\left(200 \times 200 \times 18 \mathrm{~mm}^{3}\right)$. The V-shaped slit maintains the shallowest depth $(2.0 \mathrm{~mm})$ at the top right $\mathrm{V}$-shape then goes deeper along the slit to the very bottom one $(4.8 \mathrm{~mm})$. The slit width is designed as fixed $1.0 \mathrm{~mm}$ along the pathway. It contains either seven or eight V-shaped triangles on the left or right bottom edges, respectively. The bottom edge width varies in the following decreasing order: right bottom $(167-134=33 \mathrm{~mm})$, left bottom $(150.5-119.5=31 \mathrm{~mm})$, right top bottoms $(20-15=5 \mathrm{~mm})$, and left top bottoms $(24.5-17.5=7 \mathrm{~mm})$. Accordingly, for any vertical tangent line that cuts across both sides of the triangle, one can easily assess its edge distance by the proportional principle, judging from its bottom edge and the distance to the triangular vertex, insofar as the height of each triangle is fixed at $170 \mathrm{~mm}(185-15=170 \mathrm{~mm})$. Thus, the MDD of SPECT is defined by quantifying the tangent line from the digitalized image after the SPECT image conversion from the real gamma camera scans. 


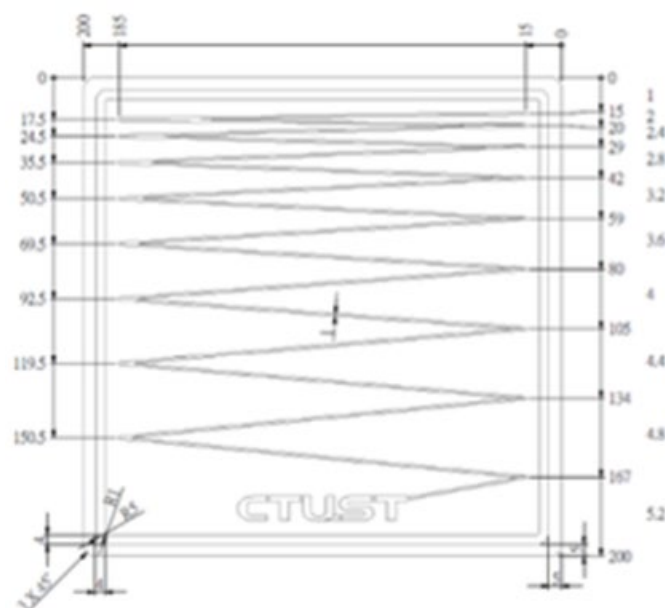

(A)

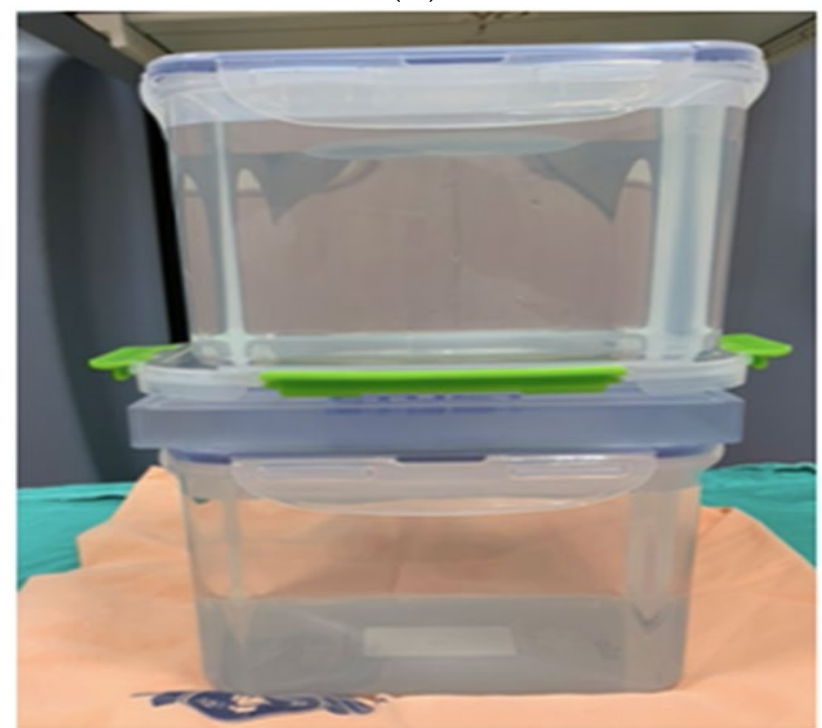

(C)
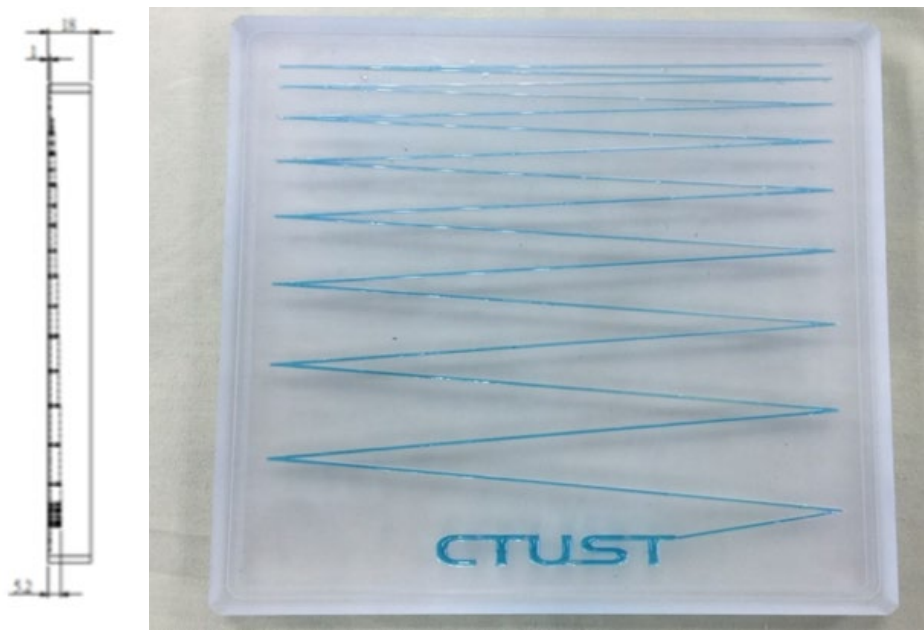

(B)

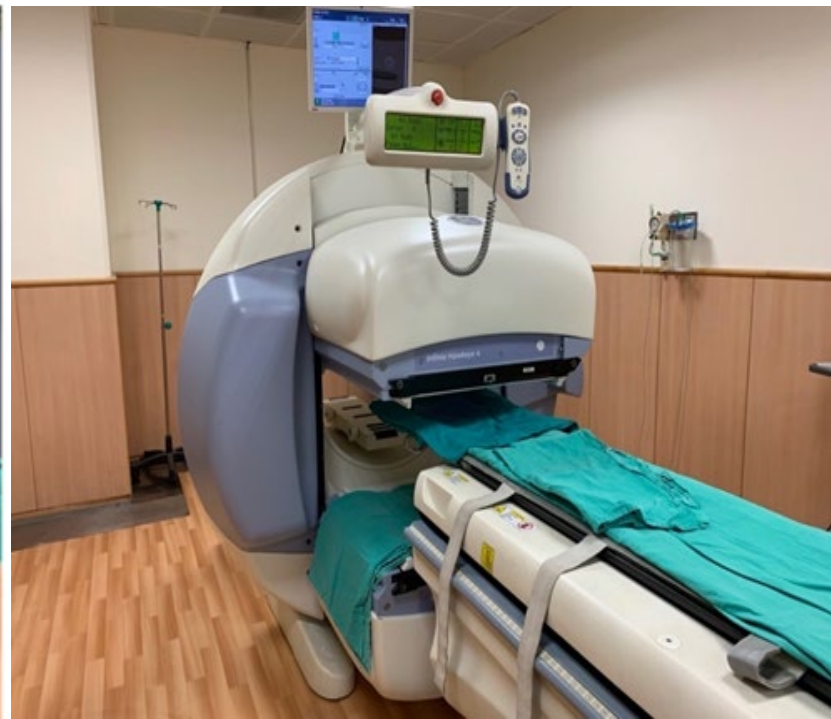

(D)

Figure 1. (A) the precise designation chart of this unique PMMA V-shaped slit gauge $(200 \times 200 \times$ $18 \mathrm{~mm}^{3}$ ). The slit width is designed as fixed $1.0 \mathrm{~mm}$ along the pathway. (B) A $7.4 \mathrm{MBq}$ Tc-99m radioactive solution is diluted to 11 c.c. and dyed with blue ink for easier identification inside the gauge. (C) The radioactive $\mathrm{V}$-shaped slit gauge was placed in the middle of two water containers. Each container was filled with $15 \mathrm{~cm}$ thick water and mixed with $0.925 \mathrm{MBq}$ Tc-99m for scanning. (D) Gamma camera GE Infinia Hawkeye 4 SPECT/CT used in this study.

In contrast to most commercially available planar line phantoms and test patterns, which implied a lead bar to create a hot slit/cold background [19], the V-shaped slit gauge provided the hot-line image with the intense-to-feeble gradation and the wide-to-narrow triangular shape for the imaging quality judgment. Figure $1 \mathrm{~B}$ shows a $7.4 \mathrm{MBq} \mathrm{Tc}-99 \mathrm{~m}$ radioactive solution, which was diluted to 11 c.c. and dyed with blue ink for easier identification; then, it was thoroughly injected into the phantom deepest part to let the solution fill in along the V-shaped pathway for the gamma camera scanning. Figure $1 \mathrm{C}$ shows the radioactive $\mathrm{V}$-shaped slit gauge, which was placed in the middle of two water containers. Each container was filled with a $15 \mathrm{~cm}$-thick water layer and mixed with $0.925 \mathrm{MBq}$ Tc-99m for scanning; this is then scanned by the gamma camera, GE Infinia Hawkeye 4 SPECT/CT, as depicted in Figure 1D. In addition, the clinical spine to soft tissue ratio in routine abdomen scan was $37.8 \pm 9.5 / 9.7 \pm 9.5$ (counts/pixel) $=3.9 \pm 3.2$ under $95 \%$ confidence level from ten randomly chosen patients in hospital, whereas the 
radioactive intensity of preset V-shaped slit gauge to the background (two water containers were summed together) was $41.8 \pm 3.9 / 11.5 \pm 0.9$ (count/pixel) $=3.6 \pm 0.4$, indicating an appropriate ratio preset in this study.

\subsection{To Rank the V-Shaped Slit Gauge}

A successful gamma camera scanned image with a superior distinguishable difference among V-shaped slits must have a fine resolution and vigorous contrast ratio to detect the fuzzy section. In doing so, three well-trained radiologists (who had over 5 years' experience in handling the radiological imaging) ranked the acquired gamma camera image in separated three days followed the double-blind criteria, because this process can significantly restrain any biased ranking from an individual radiologist [20]. Thus, the ranking score satisfied the S/N definition "higher-is-better" of the Taguchi methodology (cf. Equation (1)). Moreover, the prompt gamma camera image collected from the default acquisition system was transferred to a specific data matrix and converted by a MATLAB function (/imread) [21] to calculate the exact MDD from the derived SPECT.

\subsection{Minimum Detectable Difference and Student's t-Test Analysis}

Most researchers analyze the resolution from SPECT of gamma camera according to the individual FWHM of one peak profile, whereas in this study, the MDD was analyzed by two overlapped peak profiles collected from a tangent slice of the V-shaped slit with two neighboring peaks. Specifically, it is described as the minimum distance between two nearby peak centers via the Student's $t$-test with a constant, 1.96, which indicates that the two nearby peak centers separated far enough to create a $95 \%$ confidence level of separation [22]:

$$
\left|X_{1}-X_{2}\right| \geq 1.96 \times \sqrt{\left(\frac{F W H M_{1}}{2}\right)^{2}+\left(\frac{F W H M_{2}}{2}\right)^{2}}
$$

where $X_{1}$ and $X_{2}$ are the centers of peaks 1 and 2, respectively, while FWHM is the full width at half maximum of the specific peak. To evaluate the MDD from two nearby peaks via the unique V-shaped slit, the valley edge of two peaks was changed gradually to provide the quantitative assessment of its edge width in its original designation.

A small MDD is always preferable for the gamma camera scan image due to its superior recognizable capability in diagnosing the spine structure. As seen in Figure 2A, two peaks can be recognized as fully separated; (B) two peaks barely passed the Student's $t$-test evaluation; nevertheless, in (C), the two peaks are too close to be distinguished. Note that the principle of failing or passing the Student's $t$-test are calculated because they are completely built on the calculation results, comparing to other reports, which are mostly stood on the qualitative discrimination of radiologists and may include human factor-biased uncertainties. Therefore, the quantified MDD can provide an auxiliary tool in justifying the performing of gamma camera scanned imaging rather than radiologists' visual ranking. However, manual judgment is still essential in the preliminary sorting stage of the Taguchi analysis. 


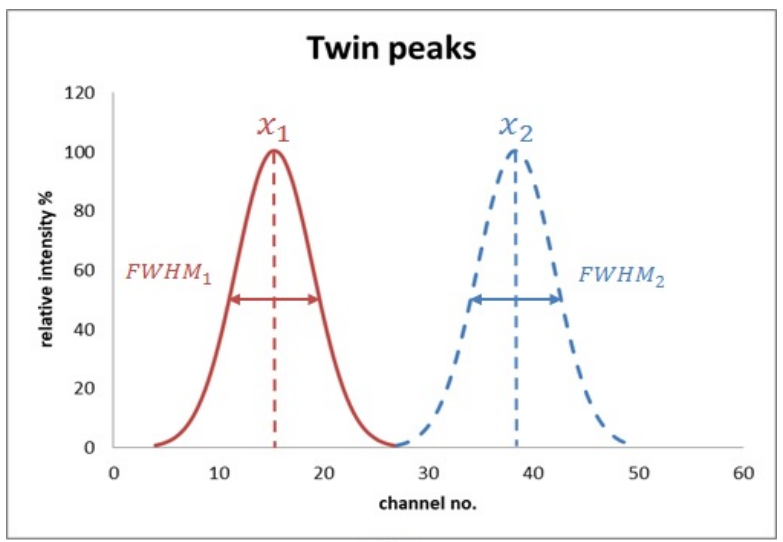

(A)

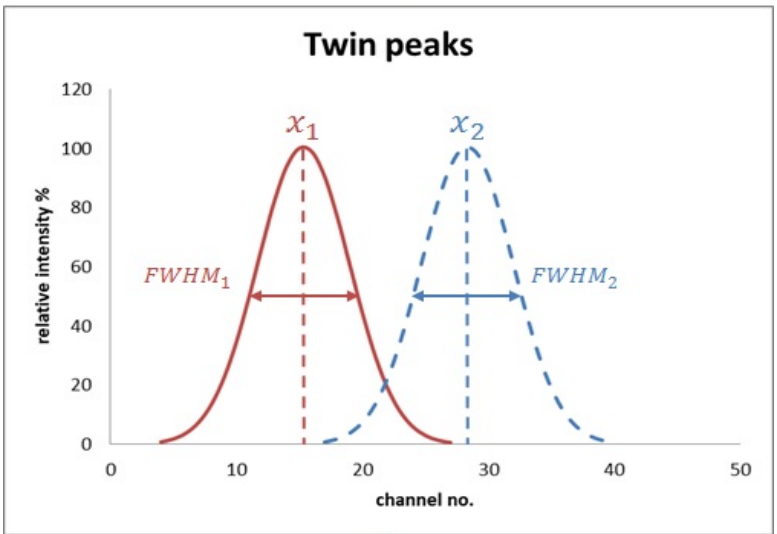

(B)

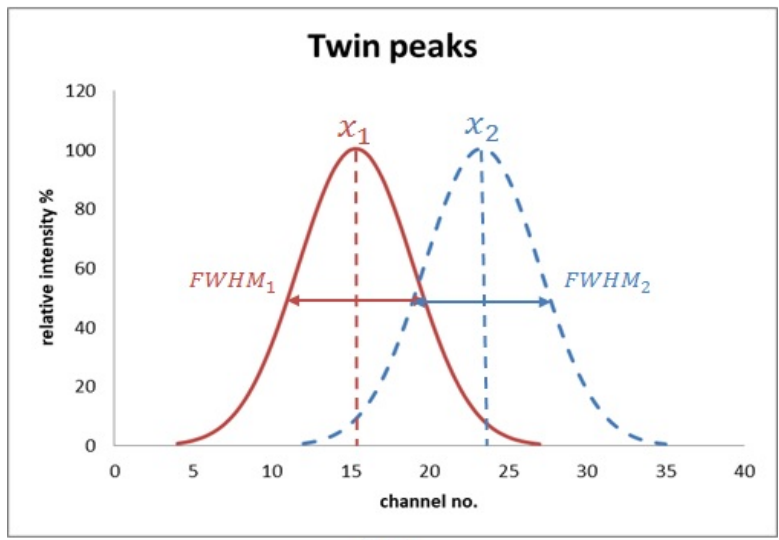

(C)

Figure 2. (A) The two peaks can be identified as wholly separated. (B) The two peaks barely passed the Student's t-test evaluation. (C) The two peaks are too close to be differentiated.

\section{Results}

\subsection{Data Analysis}

Figure 3 shows the eighteen originally scanned images from gamma camera scan, according to Taguchi's eighteen groups of factor combinations. The image quality was judged by sharpness, brightness, sensitivity, and contrast ratio altogether. The ideal images should have a strong black-and-white contrast ratio and sharp edges along the V-shaped slit. Most peaks could be easily recognized from peak one to peak six. In contrast, peak seven or eight was dependent. Thus, the precision of peak nine (cf. Figure 1A, $5 \mathrm{~mm}$ of the bottom edge) was beyond the most analytical ability of a routine gamma camera. Meanwhile, peak eight (cf. Figure 1A, $9 \mathrm{~mm}$ of the bottom edge) was the decisive part, as it would be seen from the following discussion. Table 3 shows the originally ranked grades, averages, 
standard deviations (stdev), and S/Ns derived by the three radiologists from three separate rounds (Equation (1)). The standard deviations were obtained from the ranked score $y_{i}$ in every group. Moreover, the average, stdev, and $\mathrm{S} / \mathrm{N}$ values in eighteen groups were reorganized for every specific factor. For instance, the average score of the groups $(1,4$, $7,10,13,16),(2,5,8,11,14,17)$, or $(3,6,9,12,15,18)$ implied the effects of factor $C$ (total counts, TT) at different levels $(1,2$, or 3$)$ on the performance of gamma camera-collected images (cf. Tables 1 and 2). The fish-bone plots of average, stdev, and S/N vs every specific factor of the gamma camera scan as illustrated in Figure 4, respectively. Group 2 implied the highest values within eighteen groups, as shown in Table 3. The average, stdev, and S/N values for group 2 were 16.56, 0.88, and 21.88, respectively. Accordingly, the suggested preset of six factors for the gamma camera scanned imaging system contained (A) LEHR collimator, (B) $5 \mathrm{~cm}$ DTD, (C) $150 \mathrm{k} \mathrm{TC}$, (D) $16 \%$ EW, (E) Matrix size $256 \times 256$, and (F) $1.28 \mathrm{ZOOM}$ to maintain the highest $\mathrm{S} / \mathrm{N}$ in each factor (cf. Figure $4, \mathrm{~S} / \mathrm{N}(\mathrm{dB})$ ).

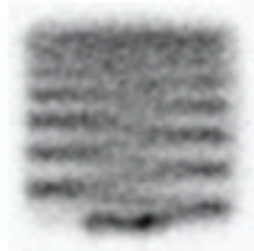

L1

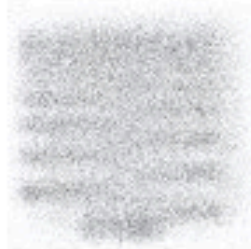

LS

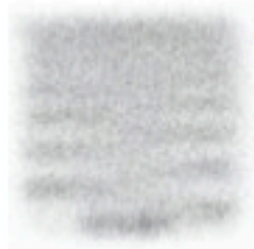

เง

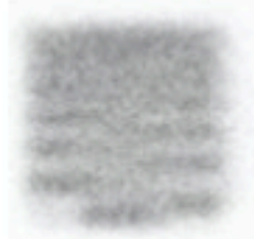

$\mathrm{L} 13$

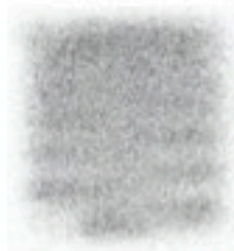

L17

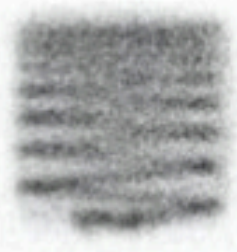

L2

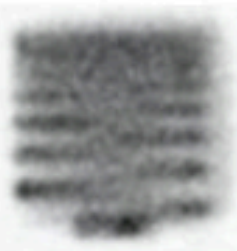

L6

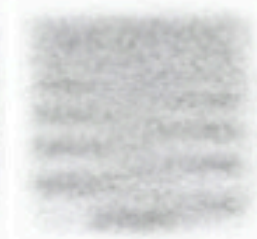

L10

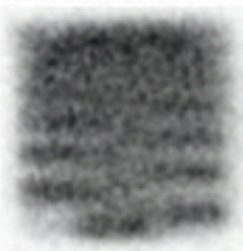

L14

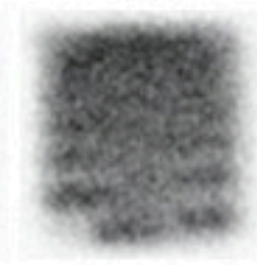

L18

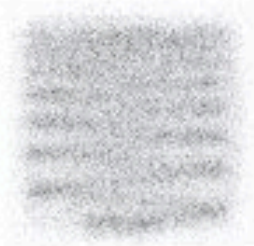

L3

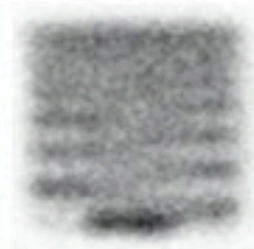

L7

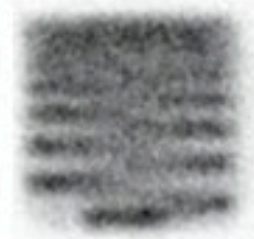

L11

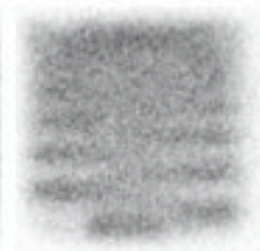

L15

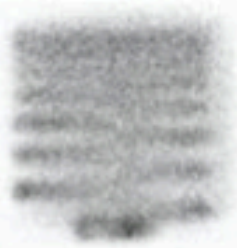

L4

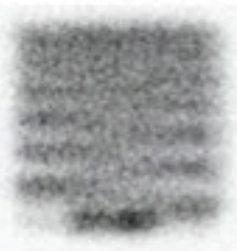

L8

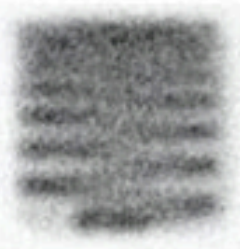

L12

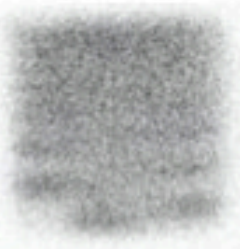

L16

Figure 3. Eighteen original scanned images from the gamma camera according to Taguchi's eighteen factor combinations. The image quality was judged by sharpness, brightness, sensitivity, and contrast ratio altogether. 
Table 3. The primary ranked scores, averages, standard deviations (stdev), and S/Ns processed by three radiologists within three separate rounds (Equation (1)). The standard deviations are derived from nine ranked grades, $y_{i}$, in each group.

\begin{tabular}{ccccccccccccc}
\hline Group & \multicolumn{3}{c}{ Radiologist-1 } & \multicolumn{3}{c}{ Radiologist-2 } & & Radiologist-3 & & Ave. & $S \boldsymbol{d}$ & S/N \\
\hline 1 & 16 & 18 & 18 & 11 & 17 & 14 & 18 & 15 & 18 & 16.11 & 2.42 & 15.78 \\
2 & 18 & 17 & 17 & 16 & 16 & 16 & 15 & 17 & 17 & 16.56 & 0.88 & 21.88 \\
3 & 13 & 16 & 16 & 18 & 13 & 18 & 12 & 18 & 14 & 15.33 & 2.40 & 15.42 \\
4 & 12 & 14 & 11 & 17 & 15 & 17 & 13 & 16 & 14 & 14.33 & 2.12 & 15.72 \\
5 & 11 & 12 & 14 & 15 & 7 & 12 & 8 & 13 & 12 & 11.56 & 2.60 & 12.35 \\
6 & 15 & 15 & 15 & 13 & 18 & 15 & 16 & 12 & 15 & 14.89 & 1.69 & 17.59 \\
7 & 6 & 5 & 3 & 8 & 8 & 7 & 3 & 9 & 8 & 6.33 & 2.24 & 8.25 \\
8 & 8 & 9 & 12 & 12 & 14 & 10 & 14 & 10 & 9 & 10.89 & 2.20 & 13.06 \\
9 & 9 & 8 & 8 & 9 & 11 & 9 & 9 & 8 & 7 & 8.67 & 1.12 & 15.24 \\
10 & 10 & 10 & 9 & 10 & 6 & 11 & 7 & 7 & 11 & 9.00 & 1.87 & 12.55 \\
11 & 14 & 11 & 10 & 14 & 9 & 8 & 10 & 11 & 10 & 10.78 & 2.05 & 13.50 \\
12 & 17 & 13 & 13 & 7 & 12 & 13 & 17 & 14 & 16 & 13.56 & 3.09 & 12.42 \\
13 & 7 & 6 & 7 & 6 & 10 & 5 & 11 & 6 & 3 & 6.78 & 2.44 & 8.21 \\
14 & 5 & 4 & 4 & 4 & 5 & 6 & 6 & 4 & 5 & 4.78 & 0.83 & 11.29 \\
15 & 4 & 7 & 5 & 5 & 4 & 4 & 4 & 5 & 6 & 4.89 & 1.05 & 10.54 \\
16 & 1 & 2 & 1 & 1 & 2 & 1 & 5 & 1 & 2 & 1.78 & 1.30 & 0.69 \\
17 & 3 & 3 & 6 & 3 & 3 & 3 & 2 & 3 & 4 & 3.33 & 1.12 & 6.94 \\
18 & 2 & 1 & 2 & 2 & 1 & 2 & 1 & 2 & 1 & 1.56 & 0.53 & 2.77 \\
Average & & & & & & & & & & $\mathbf{9 . 5}$ & $\mathbf{1 . 7 8}$ & $\mathbf{1 1 . 9 0}$ \\
\hline
\end{tabular}

\subsection{ANOVA, Analysis of Variance}

The derived confidence levels of factors attributed to the virtue of the gamma camera scan were obtained in Table 4. The importance of the dominant factors of the gamma camera scan protocol was analyzed by executing an $F$-test (cf. Equation (6)). A dominant factor was defined as providing high contribution. Therefore, factors A (collimator) of $38.5 \%$ and B (DTD) of $41 \%$ were accredited as the most affective to the imaging quality of the V-shaped slit gauge. Moreover, no factors from A to $\mathrm{F}$ could be aligned separately to assure high rank because four out of six factors (A, B, E, and F) were significant according to F-test, although factor $\mathrm{E}$ (Matrix size) or $\mathrm{F}$ (Zoom of ROI) showed a minor contribution ( $1.4 \%$ and $4.0 \%$, respectively, cf. Table 4$)$.

Table 4. The confidence levels of factors are related to the gamma camera scan protocol contributions. One factor is defined as significant if the confidence level reached $99 \%$.

\begin{tabular}{|c|c|c|c|c|c|c|c|c|}
\hline Factor & SS & DOF & Contribution & Var & F & Probability & Confidence Level & Significant * \\
\hline $\mathrm{A}$ & 1694.91 & 1 & $38.5 \%$ & 1694.9 & 463.42 & $0.0 \%$ & $100.0 \%$ & Yes \\
\hline $\mathrm{B}$ & 1784.53 & 2 & $41 \%$ & 892.3 & 243.96 & $0.0 \%$ & $100.0 \%$ & Yes \\
\hline $\mathrm{C}$ & 17.20 & 2 & $0.4 \%$ & 8.6 & 2.35 & $9.9 \%$ & $90.1 \%$ & No \\
\hline $\mathrm{D}$ & 2.68 & 2 & $0.06 \%$ & 1.3 & 0.37 & $69.4 \%$ & $30.6 \%$ & No \\
\hline $\mathrm{E}$ & 55.46 & 2 & $1.4 \%$ & 27.7 & 7.58 & $0.07 \%$ & $99.9 \%$ & Yes \\
\hline F & 176.64 & 2 & $4.0 \%$ & 88.3 & 24.15 & $0.0 \%$ & $100.0 \%$ & Yes \\
\hline Others & 110.41 & 6 & $2.5 \%$ & 18.4 & 5.03 & $0.01 \%$ & $100.0 \%$ & Yes \\
\hline Error & 526.67 & 144 & $12.1 \%$ & 3.7 & & & $S=1.91$ & \\
\hline Total & 4368.49 & 161 & $100 \%$ & & & & & \\
\hline
\end{tabular}



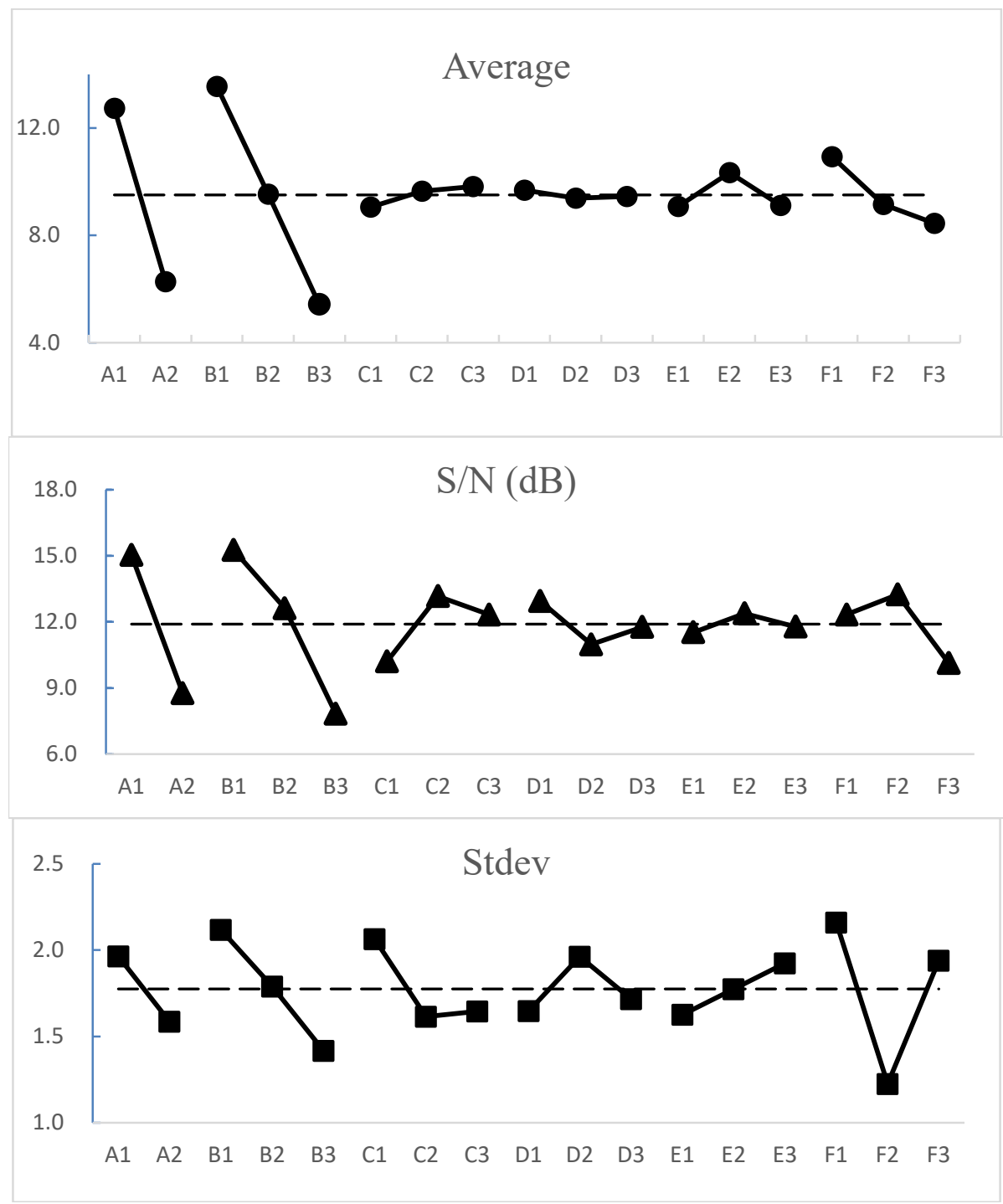

Figure 4. The fish-bone-plots of average, stdev, or $\mathrm{S} / \mathrm{N}$ versus six factors of the gamma camera scan protocol. The contribution of each factor can be analyzed and reorganized according to the Taguchi unique orthogonal array.

\section{Discussion}

\subsection{Verifying the Taguchi Recommendation by Quantified MDD}

Taguchi analysis helps efficiently optimize the gamma camera scanned imaging in the first stage according to the unique eighteen orthogonal arrays. Then, the quantified MDD is provided to verify the optimization process further. The calculated MDD can precisely quantify the performing of scanned images rather than radiologists' visual judgment in the second stage, although the professional judgment based on the double-blinded principle is practically a reliable tool. Figure 5 demonstrates the three derived SPECT and practical images from gamma camera scanned according to (A) group 2 of the original Taguchi's eighteen groups and have the highest $\mathrm{S} / \mathrm{N}$ among eighteen groups, (B) conventional preset, and (C) the combination of the highest $\mathrm{S} / \mathrm{N}$ in every specific factor (cf. Figure 4, beyond the original eighteen groups). As clearly depicted in case (A) or (B), angles 8 and 9 on the right edge are mixed (cf. Figure 1A top-right edge width was $5 \mathrm{~mm}$ ). Noteworthy is that the acquired images from a practical gamma camera were mirror-reflected, whereas in case (C), angle 9 was barely identified. Nevertheless, if checking the definition of MDD, none of the three cases can fulfill the criteria of MDD to gain a $95 \%$ confidence level from angle 8 and angle 7 (cf. Figure 1A, the bottom edge is $29-20=9 \mathrm{~mm}$ ). Thus, we focus 
on the MDD between angles 7 and 6 (bottom edge is $42-29=13 \mathrm{~mm}$ ) because the slit of this triangle has a deeper depth of $2.8 \mathrm{~mm}$ than that between angles 7 and 8 of $2.4 \mathrm{~mm}$ (cf. Figure 1A). A deeper depth can deposit comparatively more contrast media solution than a shallow one and become a bright image for calculation. Table 5 shows the correlated calculation of MDD between angles 6 and 7 of three cases in this study. The raw data of each acquired image are saved in the same data matrix. Thus, the $\mathrm{cm} /$ pixel values are the same in analyzing the specific MDD. Channel No., as adopted in Equation (7), is obtained from the SPECT of a specific case (cf. Figure 5). The second group in the original $\mathrm{L}_{18}$ has the largest MDD since it cannot separate angles 7 and 8 . In contrast, the optimal one has the smallest $\mathrm{MDD}=7.1 \mathrm{~mm}$, and the conventional one has a medium $\mathrm{MDD}=7.9 \mathrm{~mm}$ in reality. While derived MDD values may differ insignificantly, the Taguchi methodology still proves its effective capability in optimizing SPECT resolution of gamma camera images. In addition, the three different protocols of the gamma camera are rearranged in Table 6 . As demonstrated, only factors are compromised altogether, and can then minimize the MDD in reality. The quantified MDD provides reliable numerical information to help radiologists in routine quality control rather than visual inspection according to commercial phantom or line pair gauges.

Table 5. The exact derivation of the Student's $t$-test for MDD. Channel no., as adopted in Equation (7), is obtained from the SPECT of a specific case (cf. Figure 5).

\begin{tabular}{cl}
\hline Channel No. of the Derived SPECT & $\left|X_{1}-X_{2}\right| \geq 1.96 \times \sqrt{\left(\frac{F W H M_{1}}{2}\right)^{2}+\left(\frac{F W H M_{2}}{2}\right)^{2}}$ \\
\hline \multirow{2}{*}{ 2nd group } & $|354-323| \geq 1.96 \times \sqrt{\left(\frac{15}{2}\right)^{2}+\left(\frac{16}{2}\right)^{2}}$ \\
& $|31| \geq 1.96 \times \sqrt{56.25+64}$ \\
& $31.0 \geq 21.5$ \\
Conventional & $|371-340| \geq 1.96 \times \sqrt{\left(\frac{24}{2}\right)^{2}+\left(\frac{8}{2}\right)^{2}}$ \\
& $|31| \geq 1.96 \times \sqrt{144+16}$ \\
& $31.0 \geq 24.8$ \\
& $|375-342| \geq 1.96 \times \sqrt{\left(\frac{21}{2}\right)^{2}+\left(\frac{20}{2}\right)^{2}}$ \\
The Optimal & $|33| \geq 1.96 \times \sqrt{110.25+100}$ \\
& $33.0 \geq 28.4$ \\
\hline
\end{tabular}

Table 6. The MDD of $2.8 \mathrm{~mm}$ deep V-shaped slit obtained from the second group, conventional, and optimal factor settings of the gamma camera scan protocol.

\begin{tabular}{lccc}
\hline \multicolumn{1}{c}{ Factor } & 2nd Group & Conventional & Optimal \\
\hline (A) Collimator & LEHR & LEHR & LEHR \\
(B) $\quad$ Det. to Target Distance & $5 \mathrm{~cm}$ & $5 \mathrm{~cm}$ & $5 \mathrm{~cm}$ \\
(C) Total Counts & $150 \mathrm{k}$ & $165 \mathrm{k}$ & $150 \mathrm{k}$ \\
(D) Acquired Energy width & $20 \%$ & $16 \%$ & $16 \%$ \\
(E) Matrix size & $256 \times 256$ & $256 \times 256$ & $256 \times 256$ \\
(F) Zoom & 1.28 & 1.28 & 1.28 \\
MDD & $8.4 \mathrm{~mm}$ & $7.9 \mathrm{~mm}$ & $7.1 \mathrm{~mm}$ \\
\hline
\end{tabular}



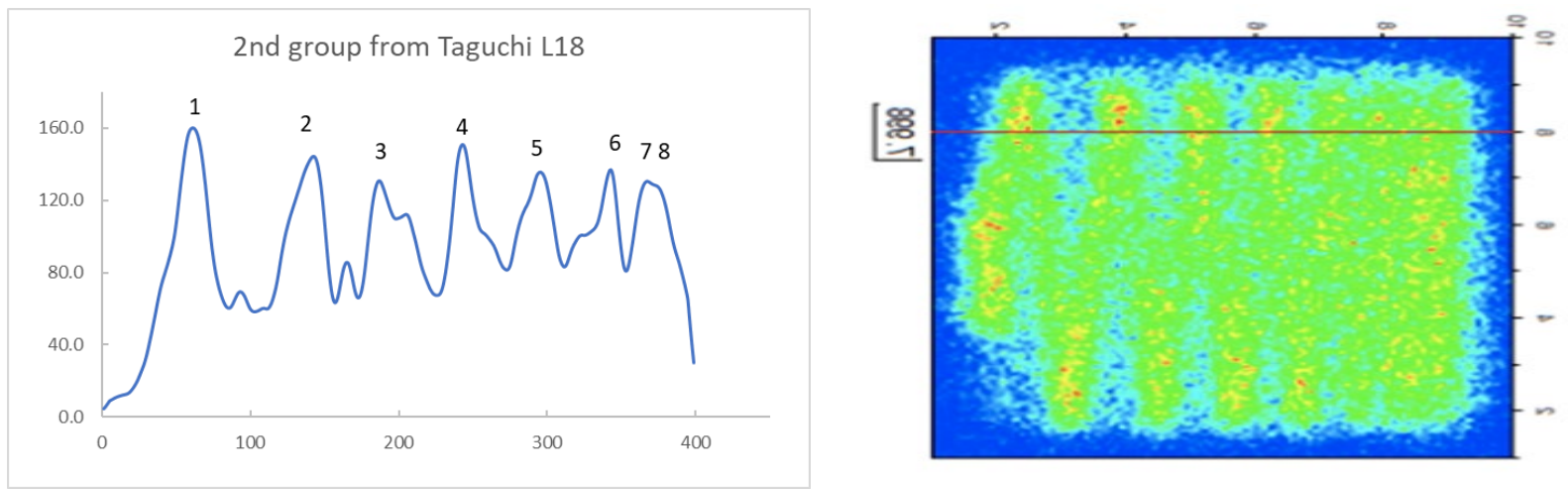

(A)
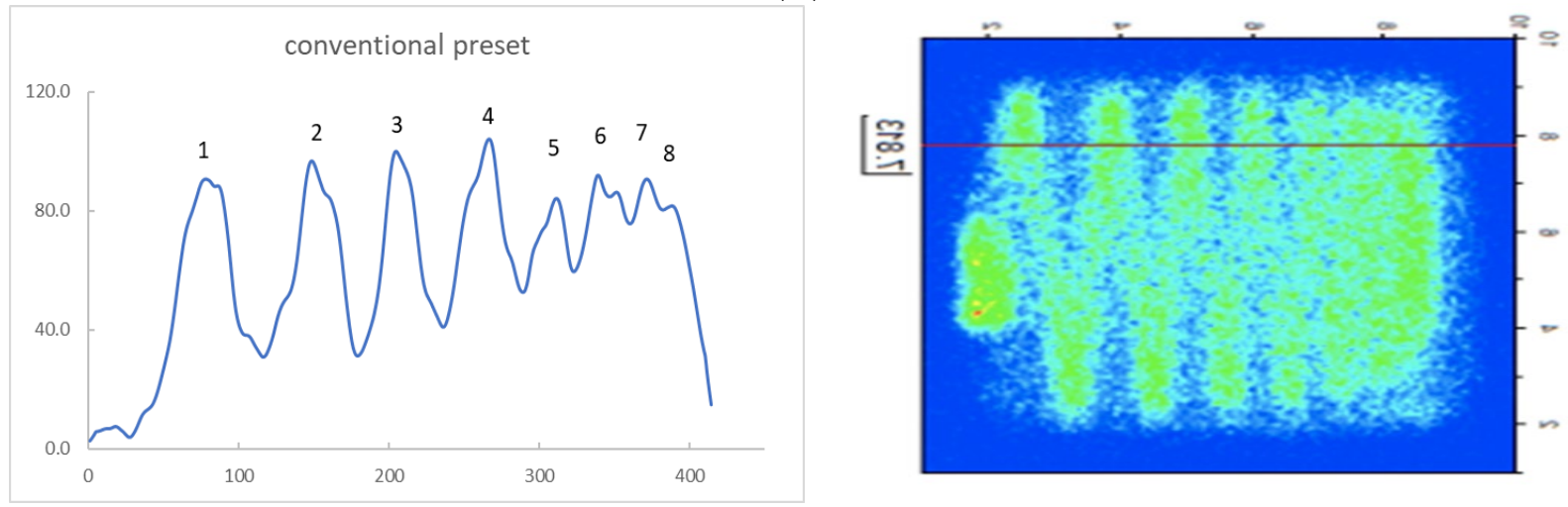

(B)
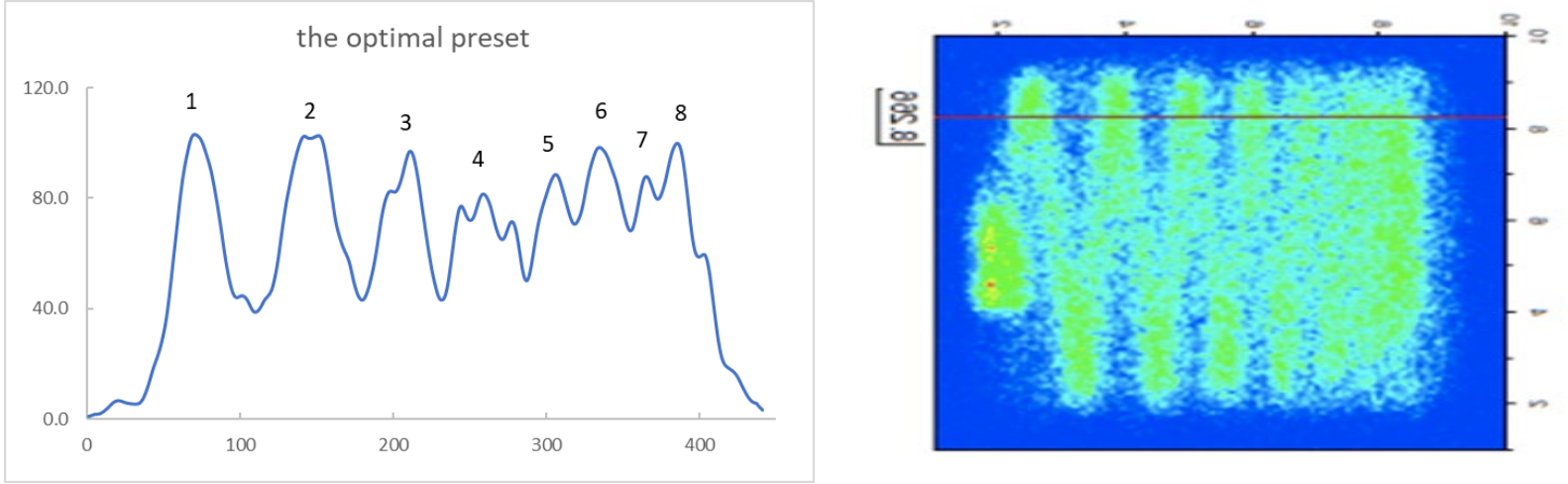

(C)

Figure 5. The three derived SPECT and original images from gamma camera scanned according to (A) group 2 of the original Taguchi's eighteen groups and have the highest $\mathrm{S} / \mathrm{N},(\mathrm{B})$ conventional preset, and $(\mathbf{C})$ the combination of the highest $\mathrm{S} / \mathrm{N}$ in every specific factor.

\subsection{Quantified MDD Superior to Line/Pair}

The quantified MDD can be transferred to the line pair $/ \mathrm{cm}(\mathrm{lp} / \mathrm{cm})$ in analyzing the gamma camera collected imaging quality, although these are defined on the basis of different scenarios in theory. The MDD is proposed to identify the minimum distance between two peaks and maintains a 95\% confidence level, yet the $\mathrm{lp} / \mathrm{cm}$ represents two lines of brightness and darkness. Furthermore, the MDD can be theoretically smaller than we derived if the confidence level is reduced from 95 to $68 \%$; then, the constant of 1.96 can be disregarded in Equation (7). As seen in Figure 6, the diagram shows the difference between MDD and $\mathrm{lp} / \mathrm{cm}$. The definition of MDD can thus be interpreted as bearing a similar width to one line pair. Thus, the number of $\mathrm{lp} / \mathrm{cm}$ approximately equals $10 \mathrm{~mm} / \mathrm{MDD}$, although MDD provides more precise information. Thus, the roughly estimated $\mathrm{lp} / \mathrm{cm}$ is $1.2,1.3$, and 1.4 for the second group, conventional, and optimal, respectively. Nevertheless, the 
$\mathrm{lp} / \mathrm{cm}$ offered only the digitized number, yet MDD is calculated based on two adjacent peaks from SPECT of the real gamma camera scanned images. In addition, Table 7 shows the various MDDs or $\mathrm{lp} / \mathrm{cm}$ from other research and this work for comparison. As clearly shown, cardiac X-ray has the finest resolution for diagnosis, whereas a gamma camera is functioned on the collection of radioactive solution inside patient's body; thus, the imaging has comparatively inferior quality among all.

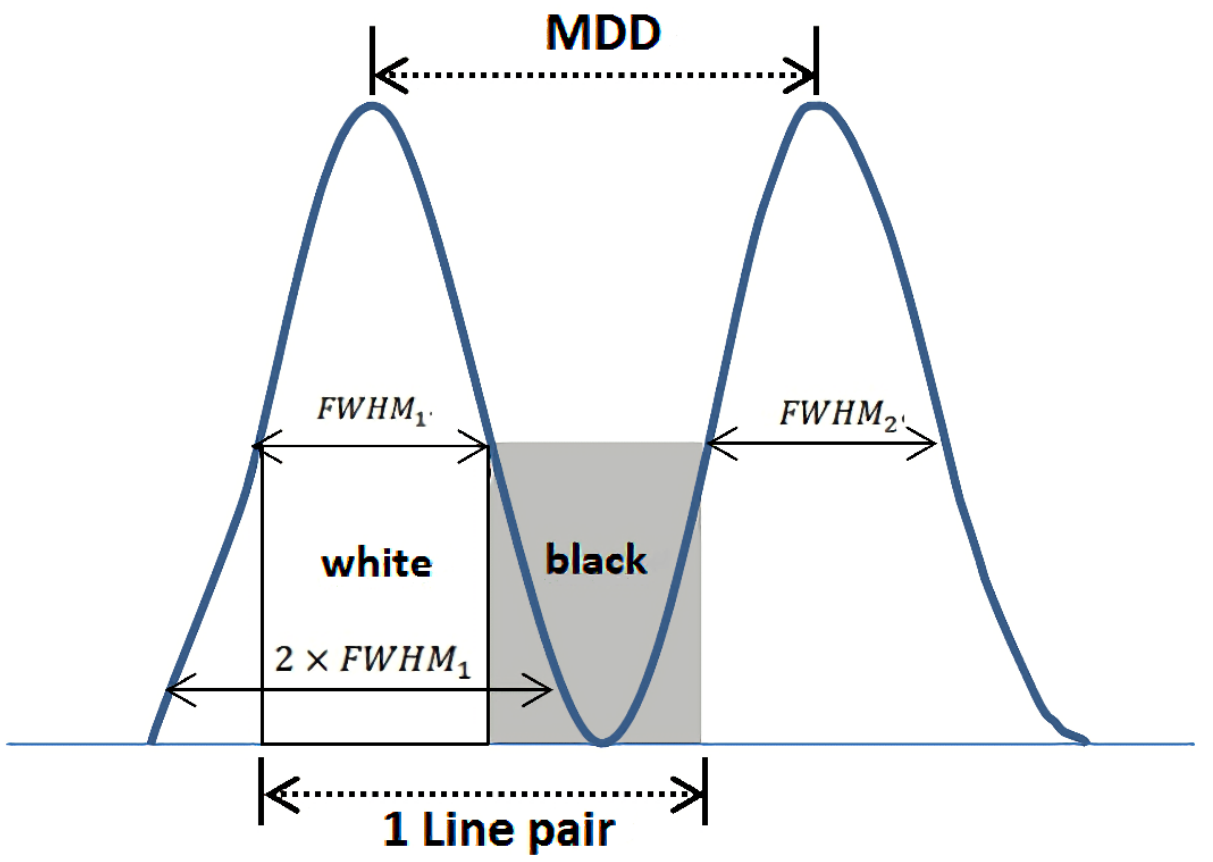

Figure 6. The diagram shows the difference between MDD and line pair $/ \mathrm{cm}$. The MDD can be interpreted as having a similar width to one line pair.

Table 7. The various MDDs or line pair/cm from other research and in this work. BPM stands for the beat per minute in the CT angiography technique.

\begin{tabular}{cccc}
\hline Reference & MDD $[\mathbf{m m}]$ or $\mathbf{l p} / \mathbf{c m}$ & Phantom & Facility \\
\hline$[12]$ & $\sim 1.0 \mathrm{lp} / \mathrm{cm}$ & Semi-quantitative plate phantom & Gamma camera \\
\hline$[13]$ & $1.45 \mathrm{~mm}$ & Simplified water phantom & Gamma camera \\
\hline$[23]$ & $0.16 \mathrm{~mm}$ & Commercial slit gauge & Cardiac X-ray \\
\hline$[24]$ & $1.43 \mathrm{~mm}$ & Indigenous slit gauge in Limb phantom & CT \\
\hline$[25]$ & $1.71 \mathrm{~mm} @ 0$ BPM & & CT angiography \\
& $2.12 \mathrm{~mm} @ 60 \mathrm{BPM}$ & Slit gauge in dynamic water phantom & \\
\hline This work & $2.44 \mathrm{~mm} @ 75 \mathrm{BPM}$ & & Gamma camera \\
\hline
\end{tabular}

\subsection{Clinical Testification of Gamma Camera Optimal Preset}

To further testify the optimal preset of the gamma camera, the scanned image was compared to the conventional preset, as shown in Figure 7. As clearly depicted, the optimal preset yielded clearer images with greater contrast than did the conventional preset. Specifically, the shadow of the ribs and pelvis could be identified, and the sacroiliac joint could also be delineated from the optimal figure. The clinical procedures had been accomplished between June to December 2021 under IRB no. B11002019 approved by the Buddhist Dalin Tuzchi Hospital. The optimization of the imaging system improved the gamma camera images for better clinical examination. 


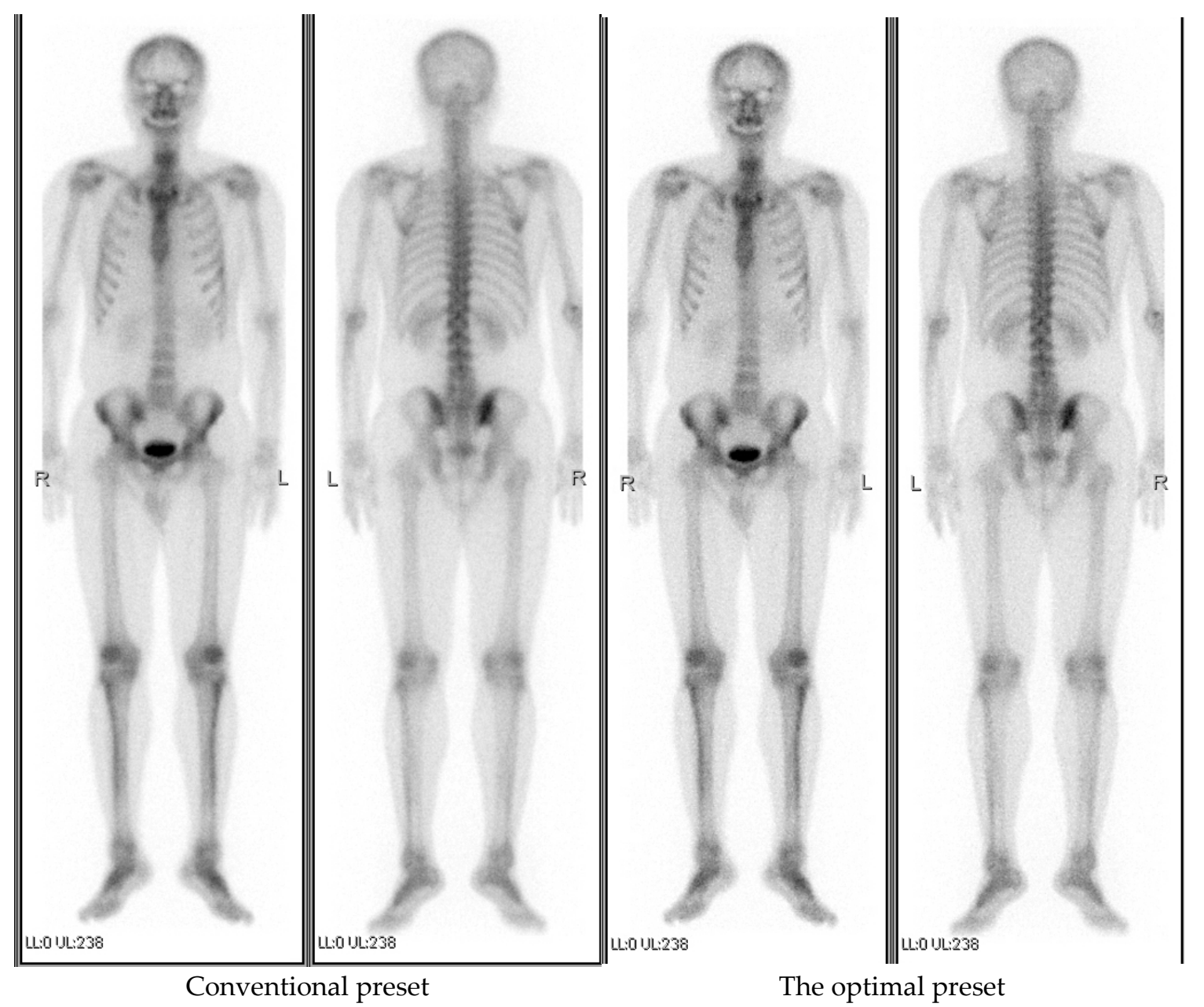

Figure 7. The optimal preset yielded clearer images with greater contrast than the conventional preset. Specifically, the shadow of the ribs and pelvis could be identified, and the sacroiliac joint could also be delineated from the optimal figure.

\section{Conclusions}

This study optimized the minimum detectable difference (MDD) of gamma camera SPECT images through an indigenous V-shaped slit gauge and the Taguchi methodology. The eighteen groups of factor combinations were organized according to Taguchi's unique orthogonal array and ranked by three well-trained radiologists to derive the signal-to-noise ratio and obtain the fish-bone plot of average, stdev, and S/N, respectively. Then, the MDD was proposed to derive further analysis of the SPECT from gamma camera scanned images. The quantified MDD was 8.4, 7.9, and $7.1 \mathrm{~mm}$ for the second group of original $\mathrm{L}_{18}$ preset, conventional, and the optimal preset, respectively, in this study. The fine MDD is beneficial in evaluating the spatial resolution than line pair $/ \mathrm{cm}$ in routine quality control. However, these two indexes were defined according to different scenarios and could be converted via a simple equation. The clinical examination proved the optimal preset of a gamma camera to have a sharp delineation. The application of MDD to justify the imaging quality in clinical nuclear medicine is expected.

Author Contributions: Conceptualization, L.-F.P. and L.-K.P.; methodology, W.-J.L.; software, C.-H.K.; validation, B.-R.P., C.-H.K. and W.-J.L.; formal analysis, W.-J.L.; investigation, B.-R.P.; resources, W.-J.L.; data curation, B.-R.P.; writing—original draft preparation, L.-F.P.; writing-review and editing, L.-K.P.; visualization, L.-F.P.; supervision, L.-K.P.; project administration, B.-R.P.; funding acquisition, L.-K.P. All authors have read and agreed to the published version of the manuscript. 
Funding: The authors highly appreciate the financial support of this study by the Ministry of Science and Technology of the Republic of China under contract No. MOST 110-2221-E-166-001 and the Taichung Armed Forces General Hospital in Taiwan (Contract No. 109A23).

Institutional Review Board Statement: The study was conducted in accordance with the Declaration of Helsinki, and approved by the Institutional Review Board of the Buddhist Dalin Tuzchi Hospital (B11002019 approved on 21 May 2021).

Informed Consent Statement: Written informed consent has been obtained from the patient(s) to publish this paper.

Data Availability Statement: Not applicable.

Conflicts of Interest: The authors declare no conflict of interest.

\section{References}

1. Yu, C.-C.; Ting, C.-Y.; Yang, M.-H.; Chan, H.-P. Comparison of irregular flux viewer system with BONENAVI version for identification of Tc-99m MDP whole body bone scan metastasis images. J. X-ray Sci. Technol. 2021, 29, 617-633. [CrossRef] [PubMed]

2. Pawar, S.U.; Dharmalingam, A.; Bhatt, B.M.; Shetye, S.S.; Ghorpade, M.K. Role of Tc-99m MDP bone scan in evaluation of osteoid osteoma at varied locations. Int. J. Res. Med. Sci. 2018, 6, 2711-2716. [CrossRef]

3. Das, D.; Das, M. Vegetation Ecology of Coastal belt of Khejuri area of Purba Medinipur District with special reference to Hijli coast, West Bengal, India. IOSR J. Pharm. 2014, 4, 56-77.

4. Archi, A.; Prafulla, J.; Nilendu, P.; Sneha, S.; Venkatesh, R. Rare splenic metastasis of renal cell carcinoma detected on 99m Tc-MDP bone scan. Indian J. Nucl. Med. 2014, 29, 60-61.

5. Araz, M.; Aras, G.; Kucuk, N.O. The role of 18F-NaF PET/CT in metastatic bone disease. J. Bone Oncol. 2015, 4, 92-97. [CrossRef]

6. Iagaru, A.; Mittra, E.; Dick, D.; Gambhir, S.S. Prospective Evaluation of 99mTc MDP Scintigraphy, 18F NaF PET/CT, and 18F FDG PET/CT for Detection of Skeletal Metastases. Mol. Imaging Biol. 2012, 14, 252-259. [CrossRef]

7. Zhang, L.; He, Q.; Zhou, T.; Zhang, B.; Li, W.; Peng, H.; Zhong, X.; Ma, L.; Zhang, R. Accurate characterization of 99mTc-MDP uptake in extraosseous neoplasm mimicking bone metastasis on whole-body bone scan: Contribution of SPECT/CT. BMC Med. Imaging 2019, 19, 44. [CrossRef]

8. Oumar, A.A.; Erçelebi, E. Assessment of an in-house phantom for the quality control of a clinical gamma camera. J. X-ray Sci. Technol. 2020, 28, 461-470. [CrossRef]

9. Robert, C.; Montémont, G.; Rebuffel, V.; Buvat, I.; Guérin, L.; Verger, L. Simulation-based evaluation and optimization of a new CdZnTe gamma-camera architecture (HiSens). Phys. Med. Biol. 2010, 55, 2709-2726. [CrossRef]

10. Hruska, C.B.; Weinmann, A.L.; O'Connor, M.K. Proof of concept for low-dose molecular breast imaging with a dual-head CZT gamma camera. Part I. Evaluation in phantoms. Med. Phys. 2012, 39, 3466-3475. [CrossRef]

11. Dickerscheid, D.; Lavalaye, J.; Romijn, L.; Habraken, J. Contrast-noise-ratio (CNR) analysis and optimisation of breast-specific gamma imaging (BSGI) acquisition protocols. EJNMMI Res. 2013, 3, 21. [CrossRef] [PubMed]

12. Yeh, D.M.; Chang, P.J.; Pan, L.K. The optimum Ga-67-citrate gamma camera imaging quality factors as first calculated and shown by the Taguchi's analysis. Hell. J. Nucl. Med. 2013, 16, 25-32. [CrossRef]

13. Kittipayak, S.; Pan, L.-K.; Chiang, F.-T.; Lin, C.-H. The Optimization of the Single Photon Emission Computed Tomography Image Quality via Taguchi Analysis: A Feasibility Study of a V-Shaped Phantom. J. Med. Imaging Health Inform. 2017, 7, 143-148. [CrossRef]

14. Chen, C.Y.; Liu, K.C.; Chen, H.H.; Pan, L.-K. Optimizing the TLD-100 readout system for various radiotherapy beam doses using the Taguchi methodology. Appl. Radiat. Isot. 2010, 68, 481-488. [CrossRef] [PubMed]

15. Pan, L.K.; Chang, B.D.; Chou, D.S. Optimization for solidification of low-level-radioactive resin using Taguchi analysis. Waste Manag. 2001, 21, 767-772. [CrossRef]

16. Roy, R.K. A Primer on the Taguchi Method, 2nd ed.; Society of Manufacturing Engineering: Southfield, MI, USA; ISBN 13:978-087263-864-8

17. Yoo, S.K.; Cotton, S.L.; Sofotasios, P.C.; Matthaiou, M.; Valkama, M.; Karagiannidis, G.K. The Fisher-Snedecor F distribution: A simple and accurate composite fading model. IEEE Commun. Lett. 2017, 21, 1661-1664. [CrossRef]

18. Nakanishi, K.; Yamamoto, S.; Kataoka, J. Performance comparison of finely channeled LYSO- and GAGG-based Si-PM gamma cameras for high-resolution SPECT. NIMA A 2017, 872, 107-111. [CrossRef]

19. Noori-Asl, M.; Sadremomtaz, A.; Bitarafan-Rajabi, A. Evaluation of three scatter correction methods based on estimation of photopeak scatter spectrum in SPECT imaging: A simulation study. Phys. Med. 2014, 30, 947-953. [CrossRef]

20. Misra, S. Randomized double blind placebo control studies, the "Gold Standard" in intervention based studies. Indian J. Sex. Transm. Dis. AIDS 2012, 33, 131-134. [CrossRef]

21. MATLAB. Matrix Laboratory Developed by MathWorks. V7.0.1.24704 (R14). 2004. Available online: http://www.mathworks, $\mathrm{com} /$ products/matlab/whatsnew.html (accessed on 4 October 2004). 
22. Kenny, D.A.; Mannetti, L.; Pierro, A.; Livi, S.; Kashy, D.A. The statistical analysis of data from small groups. J. Pers. Soc. Psychol. 2002, 83, 126-137. [CrossRef]

23. Pan, L.-K.; Wu, K.-Y.; Chen, K.-L.; Kittipayak, S. Taguchi method-based optimization of the minimum detectable difference of a cardiac X-ray imaging system using a precise line pair gauge. J. Mech. Med. Biol. 2019, 19, 1940030. [CrossRef]

24. Lee, T.-M.; Lin, C.-C.; Peng, B.-R.; Pan, L.-F. Integration of Taguchi analysis with phantom and innovative gauges: Optimization of the CT scan protocol for peripheral arterial occlusive disease (paod) syndrome. J. Mech. Med. Biol. 2020, 20, 2040005. [CrossRef]

25. Chiang, C.-Y.; Chen, Y.-H.; Pan, L.-F.; Cho, C.-C.; Peng, B.-R. Minimum detectable difference of CT angiography scans at various cardiac beats: Evaluation via a customized oblique V-shaped line gauge and pmma phantom. J. Mech. Med. Biol. 2021, $21,2140066$. [CrossRef] 